\title{
Development and validation of an algorithm to complete colonoscopy using standard endoscopes in patients with prior incomplete colonoscopy
}

\section{(ㄷ)(우우}

\author{
Authors \\ Melinda C. Rogers ${ }^{1}$, Andrew Gawron ${ }^{2}$, David Grande ${ }^{1}$, Rajesh N. Keswani ${ }^{1}$
}

Institutions

1 Gastroenterology and Hepatology, Northwestern University Feinberg School of Medicine, Chicago, Illinois, United States

2 Gastroenterology and Hepatology, University of Utah School of Medicine, Ringgold Standard Institution, Salt Lake City, Utah, United States

submitted 26.11.2016

accepted after revision 22.5.2017

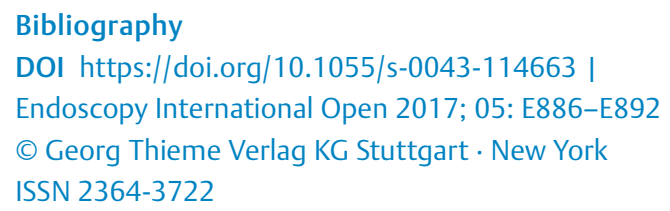

Corresponding author

Melinda Rogers, MD, Gastroenterology and Hepatology, Northwestern University Feinberg School of Medicine, 676 N St. Claire St, Suite 1400, Chicago, Illinois 606113008, United States

Fax: +1-312-695-3999

melinda.cadd@gmail.com

\section{ABSTRACT}

Background and study aims Incomplete colonoscopy may occur as a result of colon angulation (adhesions or diverticulosis), endoscope looping, or both. Specialty endo- scopes/devices have been shown to successfully complete prior incomplete colonoscopies, but may not be widely available. Radiographic or other image-based evaluations have been shown to be effective but may miss small or flat lesions, and colonoscopy is often still indicated if a large lesion is identified. The purpose of this study was to develop and validate an algorithm to determine the optimum endoscope to ensure completion of the examination in patients with prior incomplete colonoscopy.

Patients and methods This was a prospective cohort study of 175 patients with prior incomplete colonoscopy who were referred to a single endoscopist at a single academic medical center over a 3-year period from 2012 through 2015. Colonoscopy outcomes from the initial 50 patients were used to develop an algorithm to determine the optimal standard endoscope and technique to achieve cecal intubation. The algorithm was validated on the subsequent 125 patients.

Results The overall repeat colonoscopy success rate using a standard endoscope was $94 \%$. The initial standard endoscope specified by the algorithm was used and completed the colonoscopy in $90 \%$ of patients.

Conclusions This study identifies an effective strategy for completing colonoscopy in patients with prior incomplete examination, using widely available standard endoscopes and an algorithm based on patient characteristics and reasons for prior incomplete colonoscopy.

\section{Introduction}

Colonoscopy is integral to the diagnosis, prevention, and management of colon pathology. Specifically, colonoscopy is regarded by most professional societies and expert advisory groups as the mainstay of colorectal cancer screening, and is the only modality that allows both detection and removal of neoplastic polyps from the entire colon. To ensure maximal efficacy, the U.S. Multi-Society Task Force recommends that cecal intubation be achieved in at least $95 \%$ of screening colonoscopies [1]. However, studies suggest that the rate of incomplete colonoscopy may be as high as 5\%-13\% [2-4]. Incomplete colonoscopy due to inadequate bowel preparation may be successfully repeated after extended preparation, but in other cases, incomplete examination may be due to difficult anatomy that results in a tortuous colon or significant redundancy resulting in looping [5].

Recommendations following an incomplete colonoscopy are not standardized and generally reflect local expertise. Computed tomography (CT) colonography is effective but may miss small polyps or larger flat lesions [6]. The colon capsule is a newer noninvasive method to evaluate the colon and has been 
shown to be acceptable to patients [7]. Although both methods serve as viable alternatives to optical colonoscopy, these technologies are not widely available and subsequent endoscopy is needed if any abnormalities are detected. We and others have shown that novel endoscopic devices, such as single- and double-balloon enteroscopes, are effective in completing difficult colonoscopies [8-10]. Similarly, these devices may require special training and/or may not be widely available. There remain limited data on repeating colonoscopy using the standard endoscopes that are available to all gastroenterologists.

The primary aims of this prospective cohort study were to determine the success rate of repeat colonoscopy using standard endoscopes in patients referred with prior incomplete colonoscopy, and to develop a management algorithm that determines the standard endoscopes to use in patients with prior incomplete colonoscopy.

\section{Patients and methods}

\section{Study design}

This was a prospective cohort study of all patients with prior incomplete colonoscopy referred to a single endoscopist at a single academic medical center over a 3-year period (20122015). The Northwestern University Institutional Review Board approved the study. All patients provided written informed consent.

\section{Study population}

All adult patients (aged 18-90 years) with a prior incomplete colonoscopy due to anatomic reasons (tortuous and/or redundant colon) were eligible for this study. Patients whose prior colonoscopy was incomplete due only to sedation and/or poor bowel preparation were not included.

\section{Colonoscopy procedure}

All colonoscopies were performed by a single endoscopist (R.N.K.) using monitored anesthesia care (sedation administered by an anesthesiologist). Colonoscopies were initially attempted with a standard endoscope. A standard endoscope was defined as an adult (Olympus CF-H180AL; Olympus America, Center Valley, Pennsylvania, United States) or pediatric (PCF-H180AL; Olympus America) colonoscope with adjustable stiffness, or an upper endoscope with forward waterjet function (GIF-H180); Olympus America). When the colonoscopy was not successful using a standard endoscope, a single-balloon enteroscope was utilized (SIF-Q180; Olympus America). All procedures were performed with carbon dioxide insufflation. Water immersion-defined as immersion of the left colon in water during colonoscope insertion, without the use of carbon dioxide insufflation - was used in selected cases, according to the study protocol.

Patient characteristics (including age, sex, body mass index [BMI], and surgical history) were recorded on a data collection form. Additionally, a detailed colonoscopy history, including number and date of prior colonoscopies, extent reached during prior colonoscopy, endoscope(s) used in prior procedure, rea- son for incomplete colonoscopy, and use of barium enema or CT colonography, was obtained.

\section{Development of incomplete colonoscopy algorithm}

Initially, 50 consecutive patients with incomplete colonoscopy were prospectively enrolled, and repeat colonoscopy was attempted using "standard" endoscopes. An algorithm to identify the standard endoscope and technique most likely to complete the colonoscopy was developed by analyzing the outcomes of this prospective 50-patient cohort, as well as retrospective outcomes published previously by our group on the use of standard endoscopes in previously incomplete colonoscopy [5]. The algorithm proposed in our previous publication was modified for patients that were most challenging in our previous retrospective study and in the current subsequent 50-person prospective cohort: 1) patients with redundant colons and very high BMI and/or more than one prior incomplete colonoscopy; and 2) patients with tortuous colons and very low BMI. In these patients, water immersion was included in the algorithm, based on data showing that water immersion decreases looping in redundant colons and decreases patient pain during colonoscopy [11-14]. We hypothesized that water immersion would facilitate completion of colonoscopy in these particular groups because additional measures, such as abdominal pressure and patient repositioning, were unlikely to be as effective in the high BMI group, and low BMI has been associated with increased pain during colonoscopy [15].

The algorithm was then prospectively validated in 125 consecutive patients.

\section{Outcome measures}

The primary outcome of the study was a complete colonoscopy, defined as the endoscope reaching the base of the cecum and/ or the small bowel, using a standard endoscope. The secondary outcomes were: 1) complete colonoscopy using any endoscope; and 2) in the validation cohort, complete colonoscopy success rate using the initial standard endoscope determined by the algorithm.

The insertion time (total and for each individual endoscope used, if applicable), withdrawal time, and total procedure time were recorded. Total procedure time included time to change endoscopes in cases where this was required. If external pressure or change in position was required, this was recorded. A subjective level of difficulty was recorded for each procedure. All polypectomies and biopsies were performed on endoscope withdrawal.

\section{Statistical analysis}

Descriptive statistics were determined for all procedures and reported as means or medians for continuous variables and proportions for categorical variables. Comparative statistics were performed using Student's $t$ test for normally distributed variables, and Fisher's exact test to compare the association between categorical variables and outcomes. Patients were stratified by reason for prior incomplete colonoscopy: tortuous/angulated colon ("tortuous"), redundant colon due to looping ("looping”), or "both." $P$ values of $<0.05$ were considered sta- 
- Table 1 Characteristics of incomplete colonoscopy.

Time between incomplete and repeat $111(45-1641)$ colonoscopy, median (IQR), days

Extent reached, $\mathrm{n}(\%)$

\begin{tabular}{|l|l|}
\hline - Sigmoid & $58(33.1)$ \\
\hline - Descending & $21(12.0)$ \\
\hline - Transverse & $60(34.3)$ \\
\hline - Ascending & $36(20.6)$ \\
\hline
\end{tabular}

Reason for failure, $\mathrm{n}$ (\%)

- Angulation $90(51.4)$

- Redundant colon

$57(32.6)$

- Both $28(16.0)$

Multiple endoscopes used, $\mathrm{n}(\%)$ $36(20.6)$

Prior consecutive incomplete colonoscopies, $\mathrm{n}(\%)$

\begin{tabular}{|c|c|}
\hline & 1 \\
\hline & $143(81.7)$ \\
\hline 3 & $27(15.4)$ \\
\hline 5 & $4(2.3)$ \\
\hline
\end{tabular}

Reason for colonoscopy, n (\%)

- Screening $96(54.9)$

- Surveillance $35(20.0)$

- Diarrhea

$3(1.7)$

- Abdominal pain

- Family history

$12(6.9)$

- Other $24(13.7)$

$\mathrm{IQR}$, interquartile range. tistically significant. All statistical analyses were performed using SPSS version 22 (IBM Corp., Armonk, New York, USA).

\section{Results}

\section{Study population}

A total of 175 patients (69\% female) were referred to a single endoscopist for prior incomplete colonoscopy during the study period ( $\triangleright$ Table 1 ). The majority ( $84 \%$ ) of these referrals came from gastroenterologists practicing in a tertiary care setting, with the remaining coming from gastroenterologists in a community practice setting (15\%) and surgeons in a tertiary care setting ( $1 \%)$. Median age at time of referral for the entire cohort was 64 years (interquartile range [IQR] $57-69$ ). Of the 175 patients, 90 (51\%) were referred for incomplete colonoscopy due to tortuous colon, 57 (33\%) for looping due to colon redundancy, and 28 (16\%) for both (see Supplemental Table e2). During the incomplete colonoscopy, the scope did not reach beyond the descending colon in nearly half of patients (45\%). Nearly one-fifth $(18 \%)$ of patients had multiple prior incomplete colonoscopies. The majority of patients (61\%) were referred and underwent repeat colonoscopy within 1 year of incomplete colonoscopy.

Mean BMI was significantly higher in patients with incomplete colonoscopy due to looping $\left(31.0 \mathrm{~kg} / \mathrm{m}^{2}\right)$ than angulation $\left(26.3 \mathrm{~kg} \cdot \mathrm{m}^{2} ; P<0.001\right)$ ( $\triangleright$ Table 3$)$. Patients referred for incomplete colonoscopy due to tortuous colon were more likely to be female $(80 \%)$ than those referred for looping ( $46 \% ; P<0.001)$.

Abdominal pressure was utilized in $67 \%$ of cases, and did not vary between the initial study group and the validation group (66\% and $68 \%$, respectively; $P=0.80$ ). Patients were repositioned in order to facilitate completion of the colonoscopy in $19 \%$ of cases. This also did not vary significantly between the initial study group and the validation group $(8 \%$ and $24 \%$, respectively; $P=0.58)$.

- Table 3 Outcomes of repeat colonoscopy in entire cohort of incomplete colonoscopy referrals $(n=175)$.

\begin{tabular}{|c|c|c|c|c|c|}
\hline & $\begin{array}{l}\text { Tortuous } \\
(\mathrm{n}=90)\end{array}$ & $\begin{array}{l}\text { Looping } \\
(n=57)\end{array}$ & $\begin{array}{l}\text { Both } \\
(n=28)\end{array}$ & $\begin{array}{l}\text { All } \\
(n=175)\end{array}$ & $P$ value $^{1}$ \\
\hline Female sex, n (\%) & $72(80.0)$ & $26(45.6)$ & $21(75.0)$ & $119(68.0)$ & $<0.001$ \\
\hline BMI, mean (SD), kg/m² & $26.3(5.4)$ & $31.0(9.1)$ & $27(7.4)$ & $28.3(7.5)$ & $<0.001$ \\
\hline $\begin{array}{l}\text { Successful repeat colonoscopy using } \\
\text { standard endoscopes, } \mathrm{n}(\%)\end{array}$ & $88(97.8)$ & $52(91.2)$ & $25(89.3)$ & $165(94.3)$ & 0.11 \\
\hline $\begin{array}{l}\text { Successful repeat colonoscopy using any } \\
\text { endoscope, } \mathrm{n}(\%)\end{array}$ & $88(97.8)$ & $56(98.2)$ & $28(100)$ & $172(98.3)$ & $>0.99$ \\
\hline Total insertion time, median (IQR), minutes & $9.8(6.7-16.6)$ & $10.5(8.3-14.0)$ & $10.7(8.1-14.9)$ & $10.3(7.4-15.2)$ & 0.42 \\
\hline Total procedure time, median (IQR), minutes & $23.5(18.8-30.7)$ & $27.85(20.5-32.8)$ & $27.1(21.2-41.8)$ & $25.2(20.2-32.6)$ & 0.07 \\
\hline
\end{tabular}




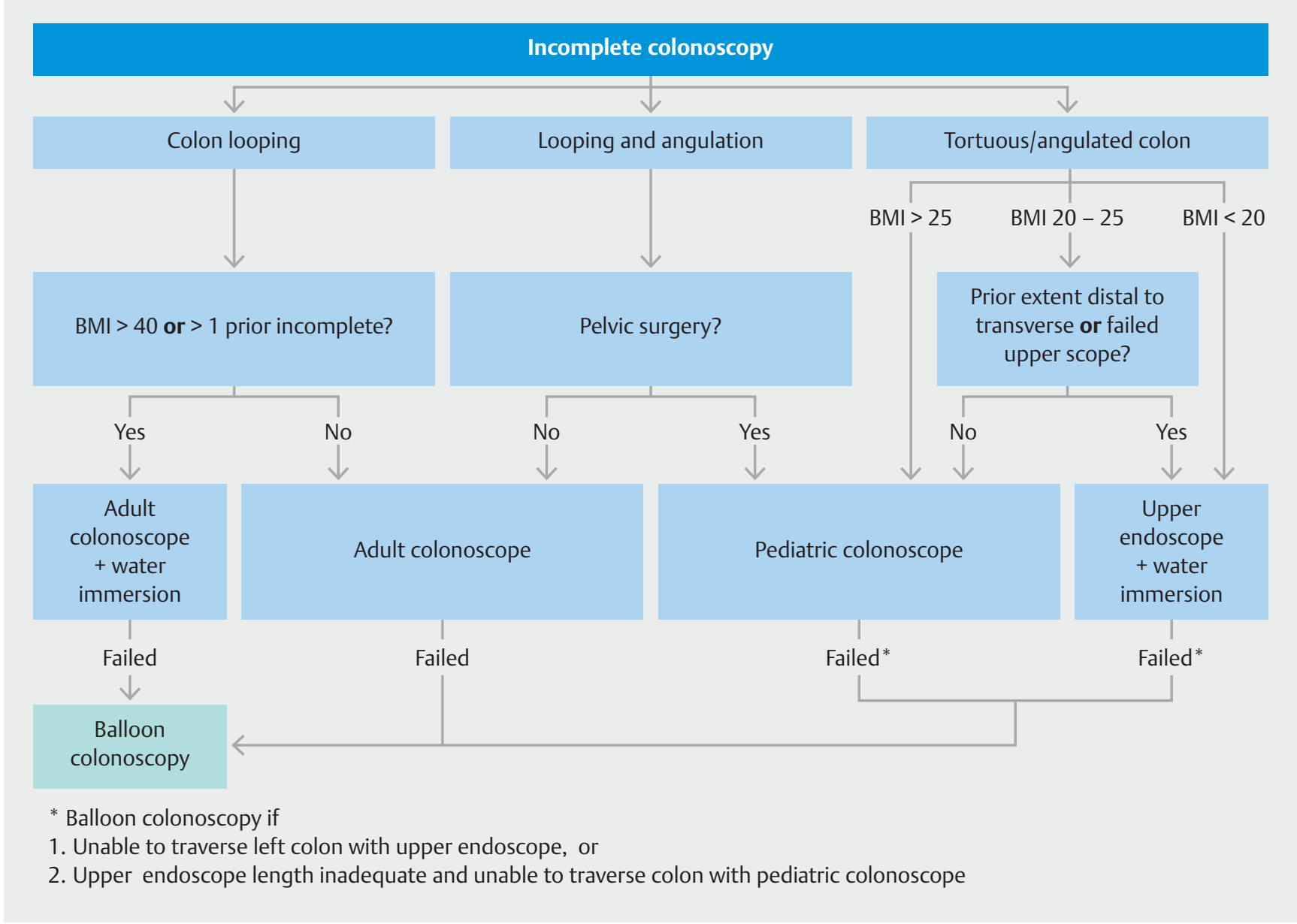

- Fig. 1 Algorithm to determine optimal endoscope and technique for successful completion of colonoscopy in patients with prior incomplete colonoscopy.

\section{Overall repeat colonoscopy success rate and outcomes}

The overall repeat colonoscopy success rate using a standard endoscope was $94 \%(n=165$; $>$ Table 3$)$. Of the 10 procedures that could not be successfully completed with a standard endoscope, 7 were completed using a single-balloon enteroscope, resulting in an overall colonoscopy completion rate of $98 \%$ (n $=172$ ) using any endoscope. Median colonoscope insertion time was 10.3 minutes [IQR 7.4-15.2] and did not differ by reason for incomplete colonoscopy.

The reason for referral, age, number of prior incomplete colonoscopies, prior surgery, presence of diverticulosis or extent reached at incomplete colonoscopy did not correlate with difficulty of repeat colonoscopy or with inability to complete colonoscopy with any endoscope. Adenomas were detected in $44 \%$ of patients. No adenocarcinomas were identified. A single adverse event occurred (transient hypoxemia requiring hospitalization).

\section{Development and validation of an incomplete colonoscopy algorithm}

Based on colonoscopy outcomes of the initial 50 patients, we developed an algorithm to determine the optimal initial standard endoscope and technique for successful cecal intubation ( $\triangleright$ Fig.1). Patient characteristics and reasons for incomplete colonoscopy did not vary between the initial study group $(n=$ $50)$ and the validation group $(n=125$; see $>$ Supplemental Table e2).

The initial standard endoscope specified by the algorithm was used and completed the colonoscopy in $90 \%$ of patients $(n=113)$; in five additional patients, use of a different standard endoscope allowed complete colonoscopy, giving a total standard colonoscope success rate of $94 \%$ in the validation cohort ( Fig. 2).

The success rate was highest when the use of an adult colonoscope with water immersion was indicated by the algorithm $(\triangleright$ Table 4$)$. Completion using the standard endoscope indicated by the algorithm was lowest in the upper endoscope with water immersion group. Of the five patients in whom colonoscopy could not be completed with the upper endoscope and water immersion, two underwent successful completion with 


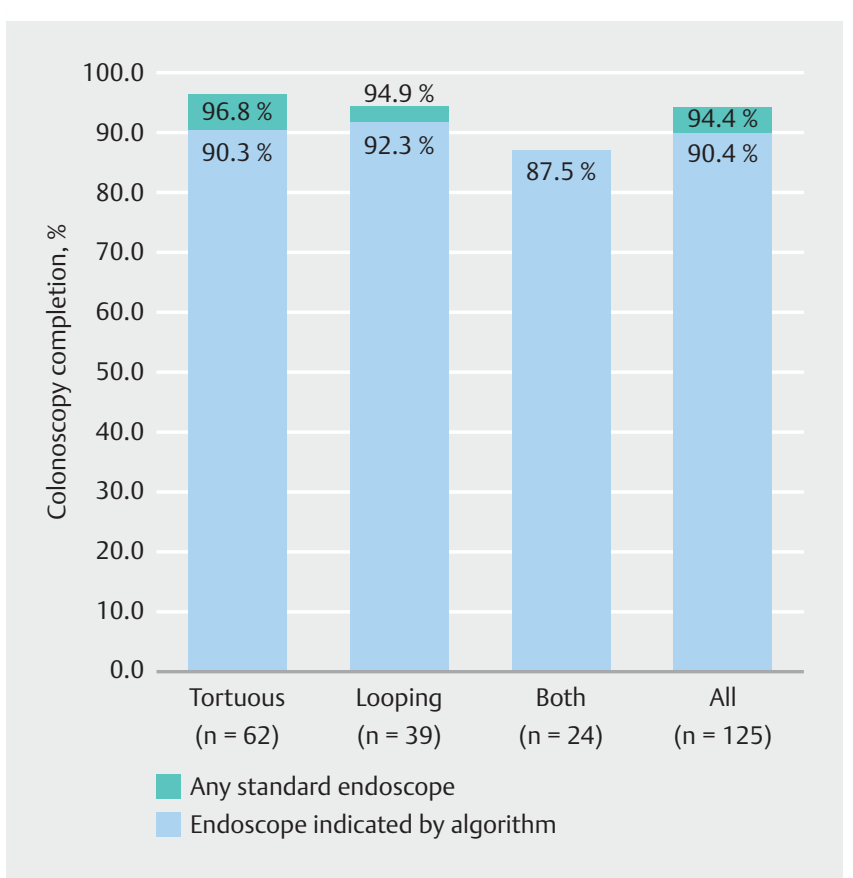

Fig. 2 Rate (\%) of colonoscopy completion using the algorithm in the validation cohort.

the pediatric colonoscope, and two were unsuccessful despite the use of a single-balloon enteroscope ( $\triangleright$ Table 4).

\section{Discussion}

We present the largest prospective study of patients undergoing repeat endoscopy following prior incomplete colonoscopy. In this study of 175 patients undergoing initial repeat endoscopy with a standard endoscope, we found that $94 \%$ of prior incomplete colonoscopies could be completed with the standard endoscope. We additionally developed and validated a management algorithm to determine the standard endoscope to use in individual patients with prior incomplete colonoscopy. Use of this algorithm led to $90 \%$ completion using the first standard endoscope selected by the algorithm. Finally, the procedure was not burdensome, with a median procedure time of 25.2 minutes and no serious adverse events.

An incomplete colonoscopy presents a clinical dilemma for healthcare providers. There is a lack of guidelines or consensus data on the optimal way to manage this cohort of patients. A variety of options have been recommended, including repeat- ing endoscopy with the use of balloon enteroscopes or pursuing radiographic or other imaging modalities. Several publications have demonstrated the safety and efficacy of using single- and double-balloon enteroscopes to achieve cecal intubation; however, availability of these devices and the expertise needed to use them safely may be limited [8-10]. Access to these specialized procedures can pose a significant barrier to patients and providers.

Double-contrast barium enema is a widely available radiographic option after incomplete colonoscopy. However, we have previously shown that the polyp detection rate of barium enema compared with optical colonoscopy is markedly lower in patients with prior incomplete colonoscopy [5]. More recently, CT colonography has been developed as an alternative approach to the evaluation of colon pathology. Two retrospective studies have evaluated the yield of this imaging modality after incomplete colonoscopy $[16,17]$. These publications demonstrated a high positive predictive value, especially for lesions 9 $\mathrm{mm}$ and larger, as well as the potential added benefit of identifying extracolonic abnormalities, particularly in patients with abdominal symptoms. There remain no data on the specificity of CT colonography, and the sensitivity diminishes for smaller lesions [16]. Furthermore, concerns regarding cumulative radiation persist. Video capsule endoscopy has an acceptable sensitivity and specificity for detecting polyps $6 \mathrm{~mm}$ and larger; however, these data were obtained specifically for the purpose of colorectal cancer screening in a population that had not failed standard optical colonoscopy [7, 18]. Critically, although these studies show promising results, they do not obviate the need for subsequent endoscopic evaluation if an abnormality is detected.

In this study, we developed a suggested treatment algorithm for patients with prior incomplete colonoscopy. Use of this algorithm resulted in a high completion rate with standard endoscopes, and a $90 \%$ success rate when using the first standard endoscope identified by the treatment algorithm. The overall $94 \%$ completion rate is comparable or superior to other published reports on the use of specialty endoscopes or devices [19]. There is increasing literature to support the yield of colonoscopy completion in this population, specifically for the purpose of colorectal cancer screening [20-22]. Our overall adenoma detection rate of $44 \%$ further supports the importance of a complete colonoscopy following incomplete colonoscopy. In the minority of patients whose colonoscopy could not be completed using a standard endoscope, a single-balloon en-

- Table 4 Outcomes of the validation cohort by endoscope indicated in the algorithm $(n=125)$.

\begin{tabular}{|l|l|l|l|}
\hline & $\begin{array}{l}\text { Success with algo- } \\
\text { rithm endoscope }\end{array}$ & $\begin{array}{l}\text { Success with different } \\
\text { standard enteroscope }\end{array}$ & $\begin{array}{l}\text { Balloon enteroscope } \\
\text { successful }\end{array}$ \\
\hline Adult colonoscope $(\mathrm{n}=37), \mathrm{n}(\%)$ & $34(91.8)$ & $1(2.7)$ & $1(2.7)$ \\
\hline Adult colonoscope + water immersion $(\mathrm{n}=13), \mathrm{n}(\%)$ & $13(100)$ & 0 & 0 \\
\hline Pediatric colonoscope $(\mathrm{n}=52), \mathrm{n}(\%)$ & $48(92.3)$ & $2(3.8)$ & $2(3.8)$ \\
\hline Upper endoscope + water immersion $(\mathrm{n}=23), \mathrm{n}(\%)$ & $18(78.3)$ & $2(8.7)$ & $1(4.3)$ \\
\hline
\end{tabular}


teroscope was used; in total, the colonoscopy was completed in $98 \%$ of patients.

There are important strengths and limitations of this study. The majority of the study population (84\%) was referred from gastroenterologists practicing in a tertiary care setting. The remainder were referred from surgeons at tertiary care centers or gastroenterologists practicing in a community setting. A strength of this study is the exclusion of patients with prior incomplete colonoscopy due to poor bowel preparation or patient discomfort, ensuring a focus only on those patients with an incomplete colonoscopy due to redundancy and tortuosity. An important limitation is that selected patients with prior incomplete colonoscopy were not referred for a repeat colonoscopy. Furthermore, as in other studies [20], all procedures were performed by a single endoscopist at a tertiary care center. We believe that this algorithm can be used as a guide for any endoscopist confronted with the dilemma of a difficult colonoscopy or a patient with prior incomplete colonoscopy. This algorithm should be validated at additional centers with other endoscopists of varied training levels and expertise. Additionally, all procedures in this study were performed with monitored anesthesia care. Although this may not be necessary in all cases, monitored anesthesia was used to eliminate the confounder of incomplete colonoscopy due to patient discomfort.

In summary, this study identifies an effective strategy for completing colonoscopy in patients with prior incomplete examination using widely available standard endoscopes. Given the high prevalence of adenomas found at repeat colonoscopy, we suggest that this algorithm is used for repeat optical colonoscopy in patients with prior incomplete colonoscopy to ensure a successful and complete examination.

\section{Competing interests}

None

\section{References}

[1] Rex DK, Bond JH, Winawer $S$ et al. Quality in the technical performance of colonoscopy and the continuous quality improvement process for colonoscopy: recommendations of the U.S. Multi-Society Task Force on Colorectal Cancer. Am J Gastroenterol 2002; 97: 1296 1308

[2] Shah HA, Paszat LF, Saskin R et al. Factors associated with incomplete colonoscopy: a population-based study. Gastroenterology 2007; 132: $2297-2303$

[3] Aslinia F, Uradomo L, Steele A et al. Quality assessment of colonoscopic cecal intubation: an analysis of 6 years of continuous practice at a university hospital. Am J Gastroenterol 2006; 101: 721 - 731
[4] Brenner H, Chang-Claude J, Jansen L et al. Role of colonoscopy and polyp characteristics in colorectal cancer after colonoscopic polyp detection: a population-based case-control study. Ann Intern Med 2012; 157: 225-232

[5] Gawron A], Veerappan A, Keswani RN. High success rate of repeat colonoscopy with standard endoscopes in patients referred for prior incomplete colonoscopy. BMC Gastroenterol 2014; 14: 56

[6] Copel L, Sosna J, Kruskal JB et al. CT colonography in 546 patients with incomplete colonoscopy. Radiology 2007; 244: 471-478

[7] Triantafyllou K, Viazis N, Tsibouris P et al. Colon capsule endoscopy is feasible to perform after incomplete colonoscopy and guides further workup in clinical practice. Gastrointest Endosc 2014; 79: 307-316

[8] Moreels TG, Macken EJ, Roth B et al. Cecal intubation rate with the double-balloon endoscope after incomplete conventional colonoscopy: a study in 45 patients. J Gastroenterol Hepatol 2010; 25: $80-83$

[9] Sulz MC, Frei R, Semadeni GM et al. The role of single-balloon colonoscopy for patients with previous incomplete standard colonoscopy: is it worth doing it? Surg Endosc 2016; 30: 1876 - 1882

[10] Yamada A, Watabe H, Takano $\mathrm{N}$ et al. Utility of single and double balloon endoscopy in patients with difficult colonoscopy: a randomized controlled trial. World J Gastroenterol 2013; 19: 4732 - 4736

[11] Asai S, Fujimoto N, Tanoue K et al. Water immersion colonoscopy facilitates straight passage of the colonoscope through the sigmoid colon without loop formation: randomized controlled trial. Dig Endosc 2015; 27: $345-353$

[12] Vemulapalli KC, Rex DK. Water immersion simplifies cecal intubation in patients with redundant colons and previous incomplete colonoscopies. Gastrointest Endosc 2012; 76: 812-817

[13] Leung FW, Amato A, Ell C et al. Water-aided colonoscopy: a systematic review. Gastrointest Endosc 2012; 76: 657-666

[14] Bourke MJ, Rex DK. Tips for better colonoscopy from two experts. Am J Gastroenterol 2012; 107: 1467-1472

[15] Park DI, Kim HJ, Park JH et al. Factors affecting abdominal pain during colonoscopy. Eur J Gastroenterol Hepatol 2007; 19: 695-699

[16] Sali L, Falchini M, Bonanomi AG et al. CT colonography after incomplete colonoscopy in subjects with positive faecal occult blood test. World J Gastroenterol 2008; 14: 4499-4504

[17] Pullens HJ, van Leeuwen MS, Laheij RJ et al. CT-colonography after incomplete colonoscopy: what is the diagnostic yield? Dis Colon Rectum 2013; 56: 593 - 599

[18] Rex DK, Adler SN, Aisenberg J et al. Accuracy of capsule colonoscopy in detecting colorectal polyps in a screening population. Gastroenterology 2015; 148: 948-957

[19] Rex DK, Chen SC, Overhiser AJ. Colonoscopy technique in consecutive patients referred for prior incomplete colonoscopy. Clin Gastroenterol Hepatol 2007; 5: 879-883

[20] Bick BL, Vemulapalli KC, Rex DK. The regional center for complex colonoscopy: yield of neoplasia in patients with prior incomplete colonoscopy. Gastrointest Endosc 2016; 83: 1239-1244

[21] Kahi C], Li X, Eckert G] et al. High colonoscopic prevalence of proximal colon serrated polyps in average-risk men and women. Gastrointest Endosc 2012; 75: 515- 520

[22] Britton EJ, Sidhu S, Geraghty J et al. The 5 -year outcome of patients having incomplete colonoscopy. Colorectal Dis 2015; 17: 298-303 
- Supplemental Table e2 Comparison of patient and procedure characteristics between initial cohort used to develop incomplete colonoscopy algorithm and validation cohort.

\begin{tabular}{|l|l|l|l|}
\hline & Initial cohort (n=50) & Validation cohort (n=125) & $\mathbf{P}$ value \\
\hline Age, median (range), years & $57(37.4-77.5)$ & $55.9(24.1-87.6)$ \\
\hline Female sex, $\mathrm{n}(\%)$ & $34(68.0)$ & $85(68.0)$ & 0.6 \\
\hline BMI, median (range), $\mathrm{kg} / \mathrm{m}^{2}$ & $35.36(15.4-55.3)$ & $34.95(16.9-53)$ \\
\hline History of prior surgery, $\mathrm{n}(\%)$ & $27(54.0)$ & $68(54.4)$ \\
\hline Number of prior incomplete colonoscopies, median (range) & $2(1-3)$ & $2(1-3)$ \\
\hline Documented reason for incomplete colonoscopy, $\mathrm{n}(\%)$ & & \\
\hline - Angulation & $28(56.0)$ & $62(49.6)$ \\
\hline - Looping & $18(36.0)$ & $39(31.2)$ \\
\hline - Both & $4(8.0)$ & $24(19.2)$ \\
\hline BMI, body mass index. & & 0.96 \\
\hline
\end{tabular}

